\title{
Suicide Rates in U.S. Presidential Election Years: 2008, 2012 and 2016
}

\author{
Paul M. Sommers \\ Department of Economics, Middlebury College, Middlebury, VT, USA \\ Email: psommers@middlebury.edu
}

How to cite this paper: Sommers, P.M. (2018) Suicide Rates in U.S. Presidential Election Years: 2008, 2012 and 2016. Open Journal of Social Sciences, 6, 33-48. https://doi.org/10.4236/jss.2018.68003

Received: June 30, 2018

Accepted: August 6, 2018

Published: August 9, 2018

Copyright $\odot 2018$ by author and Scientific Research Publishing Inc. This work is licensed under the Creative Commons Attribution International License (CC BY 4.0).

http://creativecommons.org/licenses/by/4.0/

\section{c) (i) Open Access}

\begin{abstract}
The author examines age-group specific suicide rates by state in the United States presidential election years of 2008, 2012 and 2016. For each election year, the states are divided into blue states (whose voters chose the Democratic Party presidential candidate) and red states (whose voters chose the Republican Party presidential candidate). While suicide rates have trended higher in all states, the differences in mean suicide rates in 2012 (when Democrat Barack Obama was re-elected) were significantly higher in red states than they were in blue states for every age group but adults 55 to 64 years of age. In 2016, when the Republican Party re-captured the White House late in the year, mean differences were notably higher in red states than in blue states among adults between 25 and 54 years of age.
\end{abstract}

\section{Keywords}

Suicide Rates, Presidential Elections, Red and Blue States

\section{Introduction}

In 2008, 2012 and 2016, the last three U.S. presidential election years, the highest suicide rates-18.70, 20.00 and 19.75 per 100,000 people respectively-were among American middle-aged adults, between 45 and 54 years of age (American Foundation for Suicide Prevention [1]). There is significant variation in suicide rates among states, in part due to access to firearms (the most common method of death by suicide) and in part due to low population density, notably the isolation associated with sparsely populated mostly land-locked states in the West (see, for example, Cohn [2]). Suicide has been associated with stressful economic conditions (see, for example, Hamermesh and Soss [3]). But, no one has examined whether suicide in presidential election years is related to states whose voters predominantly choose either the Republican Party (red) or Democratic 
Party (blue) presidential candidate. If the incumbent president is a Democrat, suicide rates may be higher in red states than in blue states, especially if (as in 2012) the Democratic incumbent is re-elected. The growing divide between red and blue states may manifest itself in different average suicide rates.

Barack Obama (Democrat) was the first African American elected President of the United States in 2008. He was re-elected in 2012 by defeating Republican opponent Mitt Romney. In 2016, Donald Trump (Republican), the oldest person ever elected president, defeated the Democratic presidential nominee Hillary Clinton in arguably one of the most contentious and nastiest presidential campaigns in American history. ${ }^{1}$ One might think that red states in 2012 were angrier and possibly more suicidal than blue states following Obama's re-election. In 2016, one might think that red states were angrier and possibly more suicidal than blue states until Trump's surprising victory in early November.

In this short research note, we examine state specific suicide rates over the last three presidential election years across six age groups, ranging from 20- to 24 -year-olds to adults 65 years of age or older. And, for each of the presidential election years (2008, 2012 and 2016), we propose to test for any differences in age-group specific suicide rates in blue and red states among adults over 20 years of age. Some may argue that suicide rates are invariably higher in red states only because of their geography, despite the fact that the location of "red" states has changed as several states have flipped from blue to red.

\section{Literature Review}

Levandusky and Pope [4] argued in 2011 that the degree of polarization between red and blue states was probably overstated. Based on survey data they used from 2006, the authors concluded that there was much common ground between the two groups. In 2016, however, Aisch, Pearce and Yourish [5] observed that the divide between red and blue America had become "pretty wide" and was growing. The authors speculated that the degree of polarization would continue in the 2016 presidential election. The ever-deepening divide between red and blue states before and after Donald Trump's victory in 2016 reflects strong differences in ideology. Some people (see, for example, Jacobson [6]) observed that the rift is evident in how state legislatures handle "hot-button social issues" such as gun control, abortion, and immigration. Blue states tend to approve more gun control legislation and make life easier for undocumented residents in sanctuary cities; red states have passed legislation making abortions more difficult to obtain.

No one, however, has suggested that there might be a link between the territorial separation into red and blue states across the United States and rising suicide rates. Some deeply despondent voters who complained that they had lost ${ }^{1}$ Donald J. Trump joined four other U.S. presidential winners who also lost the popular vote: John Q. Adams (1824); Rutherford B. Hayes (1876); Benjamin Harrison (1888); and George W. Bush (2000). 
control of their country might have regarded suicide as a preferable solution to waiting to vote to bring about change.

\section{Data}

The Centers for Disease Control and Prevention (CDC) in June 2018 released the latest figures (as of 2016) on age-specific death rates for suicide per 100,000 people by state. Suicide death rates by state for six age groups-20 - 24, $35-44$, 45 - 54, 55 - 64, and 65 or over-in years 2008, 2012 and 2016 were obtained from the CDC WONDER's online database for mortality [7]. In cases where the number of deaths by suicide was less than 10 (or deemed by the CDC to be "unreliable"), the CDC's figures were suppressed.

For each election year, we divide the U.S. into red and blue states. Table 1 shows age-group specific suicide rates by state and blue/red designations for the years 2008, 2012 and 2016. In 2012, two states-Indiana and North Carolina-flipped from blue to red. Four years later, in 2016, six more states-Florida, Iowa, Michigan, Ohio, Pennsylvania, and Wisconsin-flipped from blue to red. No state in either 2012 or 2016 flipped from red to blue. Presidential election maps for 2008, 2012 and 2016 [8] showing the blue/red split appear in Figures 1-3.

In 2008 and 2012, no red state and no blue state (save for Florida) was completely surrounded by rival blue/red states; in 2016, again no red state was completed surrounded by blue states, but the blue states of Minnesota and Illinois were completed surrounded by red states. The three election maps clearly show the tendency for like-minded Americans to live near one another, that is, to sort themselves geographically, a point made by Bishop [9] in 2008.

Table 2 and Table 3 summarize for blue states and red states, respectively, the number of observations for each age group, the mean, standard deviation, minimum and maximum suicide rates in 2008, 2012 and 2016. The highest average suicide rate in all three presidential election years was among adults between 45 and 54 years of age in both blue and red states. For blue states, the mean suicide rate was lowest for 20- to 24-year-olds all three years; for red states, the mean suicide rate was lowest for 20- to 24-year-olds in only 2012, while in 2008 and 2016, the mean suicide rate was lowest for the oldest age group, 65 years of age or older. Blue (red) states with the lowest suicide rates were New Jersey, Massachusetts, and New York (Nebraska, Georgia, and Louisiana); blue (red) states with the highest suicide rates were New Mexico, Nevada, and Colorado (Wyoming, Alaska, and Montana). In 2016 alone, the lowest suicide rate was 7.9 suicides per 100,000 25- to 34-year-olds in New Jersey (a blue state) and the highest suicide rate was 61.0 suicides per 100,000 20- to 24-year-olds in Alaska (a red state). Among adults 65 years of age or older in blue states, Massachusetts had the lowest suicide rate all three years; Nevada had the highest (or tied for the highest) suicide rate all three years. Among 20- to 24-year-olds (45- to 55-year-olds) in red states, Alaska (Wyoming) had the highest suicide rate all three years. 
Table 1. Age Group Suicide Rates per 100,000 by State, 2008, 2012 and $2016^{\mathrm{a}}$.

\begin{tabular}{|c|c|c|c|c|c|c|c|}
\hline \multicolumn{8}{|c|}{ Age Groups } \\
\hline State & Red/Blue ${ }^{\mathrm{b}}$ & $20-24$ & $25-34$ & $35-44$ & $45-54$ & $55-64$ & $65+$ \\
\hline \multicolumn{8}{|c|}{ Alabama } \\
\hline 2008 & red & 17.0 & 17.4 & 16.8 & 19.6 & 16.2 & 14.0 \\
\hline 2012 & red & 10.8 & 17.2 & 22.4 & 23.2 & 19.7 & 18.6 \\
\hline 2016 & red & 17.7 & 20.4 & 19.5 & 24.7 & 20.0 & 19.9 \\
\hline \multicolumn{8}{|l|}{ Alaska } \\
\hline 2008 & red & 46.0 & 31.5 & 27.6 & 29.7 & 30.8 & - \\
\hline 2012 & red & 43.3 & 36 & 30.4 & 28.2 & - & - \\
\hline 2016 & red & 61.0 & 42.3 & 38 & 23.4 & 21.0 & - \\
\hline \multicolumn{8}{|c|}{ Arizona } \\
\hline 2008 & red & 15.2 & 14.7 & 18.9 & 25.7 & 23.5 & 22.5 \\
\hline 2012 & red & 17.1 & 21.3 & 20.0 & 28.5 & 29.3 & 20.4 \\
\hline 2016 & red & 19.8 & 22.2 & 19.3 & 27.5 & 26.3 & 24.6 \\
\hline \multicolumn{8}{|c|}{ Arkansas } \\
\hline 2008 & red & 18.4 & 22.9 & 20.8 & 22.4 & 18.2 & 17.7 \\
\hline 2012 & red & 19.1 & 20.3 & 23.1 & 24.2 & 24.7 & 15.8 \\
\hline 2016 & red & 19.2 & 25.5 & 26.9 & 20.6 & 24.8 & 24 \\
\hline \multicolumn{8}{|c|}{ California } \\
\hline 2008 & blue & 9.5 & 9.3 & 12.5 & 16.8 & 17.6 & 17.1 \\
\hline 2012 & blue & 9.7 & 9.9 & 11.9 & 15.5 & 17.3 & 16.3 \\
\hline 2016 & blue & 10.8 & 11.6 & 12.2 & 15.1 & 16.7 & 16.8 \\
\hline \multicolumn{8}{|c|}{ Colorado } \\
\hline 2008 & blue & 18.6 & 15.3 & 19.2 & 29.6 & 21.7 & 21.0 \\
\hline 2012 & blue & 23.8 & 24.4 & 24.9 & 32.3 & 28.8 & 21.4 \\
\hline 2016 & blue & 25.9 & 26.4 & 26.0 & 29.4 & 27.5 & 22.9 \\
\hline \multicolumn{8}{|c|}{ Connecticut } \\
\hline 2008 & blue & 9.5 & 9.5 & 11.7 & 13.6 & 13.4 & 8.3 \\
\hline 2012 & blue & 9.2 & 11.0 & 15.9 & 16.4 & 13 & 10.3 \\
\hline 2016 & blue & 8.9 & 12.2 & 11.1 & 17.0 & 19.0 & 12.8 \\
\hline \multicolumn{8}{|c|}{ Delaware } \\
\hline 2008 & blue & - & - & - & 26.1 & - & - \\
\hline 2012 & blue & - & 23.2 & - & 23.4 & - & 14.2 \\
\hline 2016 & blue & - & - & - & 19.6 & 20.7 & 15.0 \\
\hline \multicolumn{8}{|c|}{ Florida } \\
\hline 2008 & blue & 12.7 & 15.0 & 18.7 & 24.1 & 21.6 & 18.4 \\
\hline 2012 & blue & 12.8 & 13.2 & 19.5 & 24.5 & 23.4 & 19.4 \\
\hline 2016 & red & 13.2 & 15.6 & 17.9 & 21.6 & 21.5 & 20.0 \\
\hline \multicolumn{8}{|c|}{ Georgia } \\
\hline 2008 & red & 11.7 & 11.3 & 11.9 & 16.9 & 15.3 & 15.9 \\
\hline
\end{tabular}




\section{Continued}

\begin{tabular}{|c|c|c|c|c|c|c|c|}
\hline 2012 & red & 12.2 & 15.1 & 14.1 & 17.6 & 17.3 & 16.5 \\
\hline 2016 & red & 20.3 & 16.3 & 15 & 19.9 & 19.2 & 16.1 \\
\hline \multicolumn{8}{|l|}{ Hawaii } \\
\hline 2008 & blue & - & - & 15.1 & 16.5 & - & 10.8 \\
\hline 2012 & blue & - & 15.6 & 17.2 & 19.9 & 21.0 & 13.8 \\
\hline 2016 & blue & 20.5 & 19.7 & 18.6 & 12.3 & 11.9 & 10.2 \\
\hline \multicolumn{8}{|l|}{ Idaho } \\
\hline 2008 & red & - & 12.7 & 20.2 & 24.9 & 26.2 & 28.4 \\
\hline 2012 & red & 27.6 & 18.0 & 24.2 & 33.5 & 22.9 & 19.3 \\
\hline 2016 & red & 26.8 & 15.8 & 32.5 & 39.8 & 21.1 & 27.5 \\
\hline \multicolumn{8}{|l|}{ Illinois } \\
\hline 2008 & blue & 8.7 & 10.0 & 13.5 & 14.2 & 13.5 & 11.8 \\
\hline 2012 & blue & 10.7 & 10.6 & 14.2 & 16.1 & 13.7 & 11.4 \\
\hline 2016 & blue & 12.1 & 12.0 & 15.2 & 16.2 & 15.6 & 12.5 \\
\hline \multicolumn{8}{|c|}{ Indiana } \\
\hline 2008 & blue & 9.9 & 16.0 & 19.4 & 17.0 & 16.7 & 16.5 \\
\hline 2012 & red & 14.3 & 17.0 & 21.3 & 23.1 & 18.5 & 14.8 \\
\hline 2016 & red & 20.9 & 18.8 & 21 & 24.1 & 19.3 & 16.9 \\
\hline \multicolumn{8}{|l|}{ Iowa } \\
\hline 2008 & blue & 12.6 & 15.7 & 21.2 & 18.1 & 14.7 & 12.3 \\
\hline 2012 & blue & 14.8 & 17.2 & 20.3 & 18.4 & 13.0 & 10.4 \\
\hline 2016 & red & 17.8 & 20.8 & 20.1 & 25.7 & 16.1 & 11.9 \\
\hline \multicolumn{8}{|c|}{ Kansas } \\
\hline 2008 & red & 22.7 & 15.5 & 17.1 & 15.7 & 12.3 & 13.3 \\
\hline 2012 & red & 22.0 & 22.2 & 23.0 & 28.0 & 19.9 & 18.8 \\
\hline 2016 & red & 20.8 & 27.8 & 26.2 & 24.3 & 21.2 & 16.5 \\
\hline \multicolumn{8}{|c|}{ Kentucky } \\
\hline 2008 & red & 10.1 & 16.6 & 20.5 & 22.9 & 16.3 & 19.4 \\
\hline 2012 & red & 19.7 & 20.8 & 22.4 & 24.7 & 19.7 & 18.9 \\
\hline 2016 & red & 17.3 & 21.4 & 22.6 & 26.3 & 19.6 & 19.1 \\
\hline \multicolumn{8}{|c|}{ Louisiana } \\
\hline 2008 & red & 17.1 & 15.9 & 18.7 & 15.5 & 13.8 & 14.5 \\
\hline 2012 & red & 10.8 & 15.0 & 20.2 & 17.8 & 14.8 & 15.0 \\
\hline 2016 & red & 20.2 & 19.2 & 18.6 & 19.6 & 18.7 & 16.2 \\
\hline \multicolumn{8}{|l|}{ Maine } \\
\hline 2008 & blue & - & 15.3 & 17.5 & 22.7 & 12.7 & 18.2 \\
\hline 2012 & blue & - & - & 21.0 & 29.0 & 18.9 & 15.9 \\
\hline 2016 & blue & 25.9 & 17.8 & 21.9 & 19.4 & 22.4 & 17.5 \\
\hline \multicolumn{8}{|c|}{ Maryland } \\
\hline 2008 & blue & 10.5 & 10.2 & 10.9 & 13.4 & 11.8 & 11.9 \\
\hline
\end{tabular}




\section{Continued}

\begin{tabular}{|c|c|c|c|c|c|c|c|}
\hline 2012 & blue & 12.4 & 9.6 & 12.0 & 15.1 & 14.3 & 12.7 \\
\hline 2016 & blue & 10.4 & 11.3 & 11.3 & 14.2 & 12.6 & 12.8 \\
\hline \multicolumn{8}{|c|}{ Massachusetts } \\
\hline 2008 & blue & 5.7 & 7.3 & 12.8 & 14.2 & 9.7 & 7.6 \\
\hline 2012 & blue & 10.5 & 10.2 & 13.2 & 14.7 & 12.4 & 7.0 \\
\hline 2016 & blue & 11.2 & 11.3 & 12.4 & 12.5 & 13.0 & 8.6 \\
\hline \multicolumn{8}{|c|}{ Michigan } \\
\hline 2008 & blue & 12.6 & 15.8 & 18.1 & 18.9 & 12.6 & 12.6 \\
\hline 2012 & blue & 15.5 & 16.2 & 17.4 & 18.9 & 15.3 & 12.4 \\
\hline 2016 & red & 17.3 & 16.1 & 18.1 & 19.1 & 18.2 & 14.0 \\
\hline \multicolumn{8}{|c|}{ Minnesota } \\
\hline 2008 & blue & 16.6 & 12.7 & 17.1 & 17.3 & 14.4 & 11.1 \\
\hline 2012 & blue & 12.9 & 12.1 & 19.2 & 18.7 & 17.0 & 11.2 \\
\hline 2016 & blue & 17.2 & 18.2 & 17.6 & 19.2 & 18.7 & 12.1 \\
\hline \multicolumn{8}{|c|}{ Mississippi } \\
\hline 2008 & red & 13.8 & 18.1 & 17.9 & 18 & 18.6 & 21.1 \\
\hline 2012 & red & 14.3 & 16.7 & 22.7 & 23.1 & 16.0 & 16.3 \\
\hline 2016 & red & 16.4 & 17.5 & 17.0 & 17.6 & 16.3 & 14.4 \\
\hline \multicolumn{8}{|c|}{ Missouri } \\
\hline 2008 & red & 17.3 & 15.1 & 17.5 & 21.0 & 14.1 & 15.0 \\
\hline 2012 & red & 18.2 & 19.2 & 21.1 & 22.3 & 18.7 & 17.2 \\
\hline 2016 & red & 23.0 & 26.2 & 24.5 & 27.9 & 21.0 & 18.4 \\
\hline \multicolumn{8}{|c|}{ Montana } \\
\hline 2008 & red & 29.0 & 36.1 & 26.5 & 24.2 & 22.5 & 22.1 \\
\hline 2012 & red & - & 38.8 & 34.5 & 26.4 & 32.7 & 22.1 \\
\hline 2016 & red & 27.2 & 44.4 & 36.8 & 30.2 & 28.2 & 19.5 \\
\hline \multicolumn{8}{|c|}{ Nebraska } \\
\hline 2008 & red & - & 14.1 & 12.4 & 18.5 & 11.6 & 9.6 \\
\hline 2012 & red & 15.2 & 13.9 & 16.8 & 17.6 & 17.2 & 15.2 \\
\hline 2016 & red & 20.8 & 20.6 & 16.1 & 18.3 & 15.8 & 9.8 \\
\hline \multicolumn{8}{|c|}{ Nevada } \\
\hline 2008 & blue & 23.8 & 17.9 & 23.2 & 35.0 & 31.1 & 31.4 \\
\hline 2012 & blue & 13.7 & 18.9 & 24.5 & 31.1 & 35.0 & 23.6 \\
\hline 2016 & blue & 18.3 & 22.7 & 26.8 & 30.3 & 27.3 & 37.6 \\
\hline \multicolumn{8}{|c|}{ New Hampshire } \\
\hline 2008 & blue & - & 14.6 & 21.9 & 21.5 & - & 18.7 \\
\hline 2012 & blue & - & 18.1 & 18.5 & 28.6 & 18.5 & 13.9 \\
\hline 2016 & blue & - & 17.4 & 28.3 & 22.4 & 20.4 & 23.8 \\
\hline \multicolumn{8}{|c|}{ New Jersey } \\
\hline 2008 & blue & 8.2 & 7.8 & 9.3 & 10.6 & 10.0 & 8.7 \\
\hline
\end{tabular}




\section{Continued}

\begin{tabular}{|c|c|c|c|c|c|c|c|}
\hline 2012 & blue & 12.1 & 8.6 & 10.8 & 11.0 & 10.7 & 7.6 \\
\hline 2016 & blue & 8.8 & 7.9 & 8.5 & 12.3 & 11.0 & 9.2 \\
\hline \multicolumn{8}{|c|}{ New Mexico } \\
\hline 2008 & blue & 25.5 & 23.0 & 27.0 & 35.1 & 19.6 & 24.8 \\
\hline 2012 & blue & 22 & 24.2 & 31.0 & 34.0 & 25.4 & 23.4 \\
\hline 2016 & blue & 25.4 & 30.4 & 34.2 & 30.6 & 26.7 & 27.5 \\
\hline \multicolumn{8}{|c|}{ New York } \\
\hline 2008 & blue & 7.3 & 7.6 & 10.3 & 11.7 & 9.3 & 9.4 \\
\hline 2012 & blue & 8.9 & 9.2 & 10 & 14.1 & 12.4 & 10.2 \\
\hline 2016 & blue & 9.6 & 8.5 & 10.8 & 12.2 & 12.1 & 9.9 \\
\hline \multicolumn{8}{|c|}{ North Carolina } \\
\hline 2008 & blue & 14.4 & 14.9 & 17.4 & 19.2 & 16.0 & 15.0 \\
\hline 2012 & red & 13.8 & 15.3 & 14 & 23.0 & 18.4 & 16.2 \\
\hline 2016 & red & 15.9 & 14.2 & 17.7 & 18.7 & 19.4 & 16.1 \\
\hline \multicolumn{8}{|c|}{ North Dakota } \\
\hline 2008 & red & - & - & - & - & - & - \\
\hline 2012 & red & - & - & 26.0 & 23.6 & - & - \\
\hline 2016 & red & - & 25.5 & 25.5 & 35.1 & - & - \\
\hline \multicolumn{8}{|l|}{ Ohio } \\
\hline 2008 & blue & 13.7 & 16.0 & 19.0 & 17.8 & 13.8 & 12.0 \\
\hline 2012 & blue & 13.9 & 16.5 & 18.1 & 19.7 & 17.3 & 15.1 \\
\hline 2016 & red & 16.2 & 20.1 & 18.2 & 19.7 & 20.4 & 16.2 \\
\hline \multicolumn{8}{|c|}{ Oklahoma } \\
\hline 2008 & red & 16.4 & 19.4 & 22.6 & 26.0 & 18.2 & 17.5 \\
\hline 2012 & red & 22.7 & 23 & 26.8 & 20.3 & 22.7 & 18.9 \\
\hline 2016 & red & 30.1 & 28.4 & 29.4 & 29.8 & 26.4 & 21.2 \\
\hline \multicolumn{8}{|l|}{ Oregon } \\
\hline 2008 & blue & 13.9 & 14.7 & 20.5 & 25.9 & 21.4 & 16.9 \\
\hline 2012 & blue & 13.9 & 20.6 & 20.0 & 36.1 & 21.4 & 23.6 \\
\hline 2016 & blue & 22.4 & 16.5 & 22.7 & 27.7 & 25 & 25.8 \\
\hline \multicolumn{8}{|c|}{ Pennsylvania } \\
\hline 2008 & blue & 12.9 & 15.6 & 17.1 & 19.8 & 14.3 & 12.7 \\
\hline 2012 & blue & 15.4 & 15.4 & 17.8 & 19.6 & 17.0 & 12.8 \\
\hline 2016 & red & 17.2 & 17.5 & 19.6 & 23.6 & 20.7 & 16.2 \\
\hline \multicolumn{8}{|c|}{ Rhode Island } \\
\hline 2008 & blue & - & - & 17.9 & 22.2 & 16.3 & - \\
\hline 2012 & blue & - & - & - & 20.9 & - & - \\
\hline 2016 & blue & - & - & 17.1 & 20.4 & 19.7 & - \\
\hline \multicolumn{8}{|c|}{ South Carolina } \\
\hline 2008 & red & 16.9 & 11.9 & 14.1 & 19.6 & 18.5 & 15.2 \\
\hline 2012 & red & 13.2 & 16.6 & 16.4 & 23.9 & 19.6 & 17.8 \\
\hline 2016 & red & 21 & 18.3 & 18.9 & 25.5 & 22.3 & 19 \\
\hline \multicolumn{8}{|c|}{ South Dakota } \\
\hline 2008 & red & - & - & 22.0 & 18.8 & - & - \\
\hline
\end{tabular}




\section{Continued}

\begin{tabular}{|c|c|c|c|c|c|c|c|}
\hline 2012 & red & - & 23.6 & - & 29.3 & 19.7 & - \\
\hline 2016 & red & - & 24.9 & 39.6 & 22.5 & - & - \\
\hline \multicolumn{8}{|c|}{ Tennessee } \\
\hline 2008 & red & 17.4 & 17.2 & 23.1 & 23.3 & 24.4 & 16.4 \\
\hline 2012 & red & 12.1 & 17.1 & 19 & 24.4 & 20.4 & 20.4 \\
\hline 2016 & red & 14.9 & 20.6 & 22.4 & 25 & 20.7 & 19.5 \\
\hline \multicolumn{8}{|l|}{ Texas } \\
\hline 2008 & red & 11.6 & 12.8 & 13.8 & 17 & 14.4 & 15.2 \\
\hline 2012 & red & 14.1 & 13.7 & 14.7 & 17.6 & 16.7 & 16.7 \\
\hline 2016 & red & 15.8 & 16.3 & 15.2 & 17.8 & 17 & 15.7 \\
\hline \multicolumn{8}{|l|}{ Utah } \\
\hline 2008 & red & 22.3 & 16.8 & 20.8 & 30.4 & 22.6 & 13.6 \\
\hline 2012 & red & 19.9 & 25.6 & 28.6 & 40.2 & 26 & 20.6 \\
\hline 2016 & red & 23.7 & 27.2 & 31.4 & 35 & 26.1 & 22.4 \\
\hline \multicolumn{8}{|l|}{ Vermont } \\
\hline 2008 & blue & - & - & - & 26.1 & - & - \\
\hline 2012 & blue & - & - & - & 23.5 & - & - \\
\hline 2016 & blue & - & 30.3 & - & 24.3 & 25.8 & 22.1 \\
\hline \multicolumn{8}{|l|}{ Virginia } \\
\hline 2008 & blue & 13.1 & 13.1 & 15.1 & 17.9 & 15.4 & 16.7 \\
\hline 2012 & blue & 14 & 15 & 17.4 & 18.2 & 17 & 16.8 \\
\hline 2016 & blue & 18 & 17.3 & 15.1 & 16.8 & 18.4 & 18.7 \\
\hline \multicolumn{8}{|c|}{ Washington } \\
\hline 2008 & blue & 15.1 & 14.5 & 17.4 & 21.5 & 18 & 17.9 \\
\hline 2012 & blue & 16.3 & 18.1 & 17 & 22.2 & 20.8 & 18.2 \\
\hline 2016 & blue & 20.3 & 17.8 & 17.3 & 20.6 & 23.4 & 19.1 \\
\hline \multicolumn{8}{|c|}{ West Virginia } \\
\hline 2008 & red & 18.2 & 17.2 & 22.7 & 18.6 & 15.2 & 16.4 \\
\hline 2012 & red & 21.7 & 24.1 & 25.8 & 26.5 & 21.3 & 13.8 \\
\hline 2016 & red & 18.7 & 27.4 & 35.9 & 22.6 & 22.9 & 20.7 \\
\hline \multicolumn{8}{|c|}{ Wisconsin } \\
\hline 2008 & blue & 12.8 & 17 & 19.2 & 20.2 & 19.8 & 12.3 \\
\hline 2012 & blue & 15.9 & 17.3 & 16.6 & 19.5 & 15.4 & 11 \\
\hline 2016 & red & 19.4 & 18.4 & 20.8 & 21.7 & 17.6 & 14.5 \\
\hline \multicolumn{8}{|c|}{ Wyoming } \\
\hline 2008 & red & - & 29.4 & 34.4 & 30.6 & - & - \\
\hline 2012 & red & - & 45.6 & 39.6 & 45.6 & 31.7 & 31.8 \\
\hline 2016 & red & - & 25.6 & 39.3 & 40.5 & - & 36.4 \\
\hline
\end{tabular}

${ }^{\mathrm{a} D a t a}$ sources: Suicide rates per 100,000 population by state for residents 20 to 24 years of age in years 2008 , 2012, 2016 are from: https://wonder.cdc.gov/controller/saved/D76/D38F594; suicide rates by state for residents between 25 and 64 years of age by ten-year age groups in years 2008, 2012, 2016 are from: https://wonder.cdc.gov/controller/saved/D76/D38F595; and suicide rates by state for residents 65 years of age or over in years 2008, 2012, 2016 are from: https://wonder.cdc.gov/controller/saved/D76/D38F596. ${ }^{b}$ Blue states (whose electoral votes or majority of electoral votes went to the Democratic presidential candidate) and red states (whose electoral votes went to the Republican presidential candidate) are from https://www.270towin.com/maps/2008-election-state-winners, https://www.270towin.com/maps/2012-election-state-winners, and https://www.270towin.com/maps/2016-election-state-winners. 
Table 2. Age group suicide rates, blue states, 2008, 2012 and 2016, Summary statistics.

\begin{tabular}{|c|c|c|c|c|c|c|}
\hline Age Groups & $20-24$ & $25-34$ & $35-44$ & $45-54$ & $55-64$ & $65+$ \\
\hline \multicolumn{7}{|l|}{2008} \\
\hline Observations & 22 & 24 & 26 & 28 & 24 & 25 \\
\hline Mean & 13.07 & 13.7 & 17.04 & 20.25 & 16.06 & 14.96 \\
\hline Std. Dev. & 4.84 & 3.76 & 4.21 & 6.23 & 4.85 & 5.46 \\
\hline \multirow[t]{2}{*}{ Minimum } & 5.7 & 7.3 & 9.3 & 10.6 & 9.3 & 7.6 \\
\hline & Massachusetts & Massachusetts & $\begin{array}{l}\text { New } \\
\text { Jersey }\end{array}$ & New Jersey & New York & Massachusetts \\
\hline \multirow[t]{2}{*}{ Maximum } & 25.5 & 23 & 27 & 35.1 & 31.1 & 31.4 \\
\hline & New Mexico & New Mexico & $\begin{array}{c}\text { New } \\
\text { Mexico }\end{array}$ & $\begin{array}{c}\text { New } \\
\text { Mexico }\end{array}$ & Nevada & Nevada \\
\hline \multicolumn{7}{|l|}{2012} \\
\hline Observations & 20 & 23 & 23 & 26 & 23 & 24 \\
\hline Mean & 13.92 & 15.44 & 17.76 & 21.59 & 18.22 & 14.69 \\
\hline Std. Dev. & 3.79 & 4.84 & 4.84 & 6.62 & 5.78 & 4.85 \\
\hline \multirow[t]{2}{*}{ Minimum } & 8.9 & 8.6 & 10 & 11 & 10.7 & 7 \\
\hline & New York & New Jersey & $\begin{array}{l}\text { New } \\
\text { York }\end{array}$ & New Jersey & New Jersey & Massachusetts \\
\hline \multirow[t]{2}{*}{ Maximum } & 23.8 & 24.4 & 31 & 36.1 & 35 & 23.6 \\
\hline & Colorado & Colorado & $\begin{array}{c}\text { New } \\
\text { Mexico }\end{array}$ & Oregon & Nevada & $\begin{array}{l}\text { Nevada, } \\
\text { Oregon }\end{array}$ \\
\hline \multicolumn{7}{|l|}{2016} \\
\hline Observations & 16 & 18 & 18 & 20 & 20 & 19 \\
\hline Mean & 16.61 & 17.18 & 18.17 & 19.63 & 19.4 & 17.63 \\
\hline Std. Dev. & 6.37 & 6.79 & 7.13 & 6.14 & 5.49 & 7.57 \\
\hline \multirow[t]{2}{*}{ Minimum } & 8.8 & 7.9 & 8.5 & 12.2 & 11 & 8.6 \\
\hline & New Jersey & New Jersey & $\begin{array}{l}\text { New } \\
\text { Jersey }\end{array}$ & New York & New Jersey & Massachusetts \\
\hline \multirow[t]{2}{*}{ Maximum } & 25.9 & 30.4 & 34.2 & 30.6 & 27.5 & 37.6 \\
\hline & $\begin{array}{l}\text { Colorado, } \\
\text { Maine }\end{array}$ & New Mexico & $\begin{array}{c}\text { New } \\
\text { Mexico }\end{array}$ & $\begin{array}{c}\text { New } \\
\text { Mexico }\end{array}$ & Colorado & Nevada \\
\hline
\end{tabular}

Suicide rates have increased for all six age groups in blue as well as in red states since 2008. Among blue states, the percentage increase in the average suicide rate between 2008 and 2016 was highest (27.1 percent, from 13.07 to 16.61) for the youngest adults aged 20 to 24 . Among red states, the corresponding percentage increase was highest (22.8 percent, from 18.33 to 22.51 ) for adults between 25 and 34 years of age. The average suicide rate between 2008 and 2016 actually fell 3.1 percent (from 20.25 to 19.63 ) in blue states among adults between 45 and 54 years of age.

\section{Methodology}

In order to determine whether or not average suicide rates by age group have changed markedly between presidential election years for 1) blue states, 2) red 
Table 3. Age group suicide rates, red states, 2008, 2012 and 2016, summary statistics.

\begin{tabular}{|c|c|c|c|c|c|c|}
\hline Age Groups & $20-24$ & $25-34$ & $35-44$ & $45-54$ & $55-64$ & $65+$ \\
\hline \multicolumn{7}{|l|}{2008} \\
\hline Observations & 17 & 20 & 21 & 21 & 19 & 18 \\
\hline Mean & 18.89 & 18.33 & 20.01 & 21.87 & 18.56 & 17.1 \\
\hline Std. Dev. & 8.32 & 6.69 & 5.32 & 4.71 & 5.16 & 4.32 \\
\hline \multirow[t]{2}{*}{ Minimum } & 10.1 & 11.3 & 11.9 & 15.5 & 11.6 & 9.6 \\
\hline & Kentucky & Georgia & Georgia & Louisiana & Nebraska & Nebraska \\
\hline \multirow[t]{2}{*}{ Maximum } & 46 & 36.1 & 34.4 & 30.6 & 30.8 & 28.4 \\
\hline & Alaska & Montana & Wyoming & Wyoming & Alaska & Idaho \\
\hline \multicolumn{7}{|l|}{2012} \\
\hline Observations & 20 & 23 & 23 & 24 & 22 & 21 \\
\hline Mean & 18.11 & 21.57 & 22.92 & 25.53 & 21.27 & 18.34 \\
\hline Std. Dev. & 7.45 & 8.22 & 6.32 & 6.72 & 4.88 & 3.79 \\
\hline \multirow[t]{2}{*}{ Minimum } & 10.8 & 13.7 & 14 & 17.6 & 14.8 & 13.8 \\
\hline & $\begin{array}{l}\text { Alabama, } \\
\text { Louisiana }\end{array}$ & Texas & $\begin{array}{l}\text { North } \\
\text { Carolina }\end{array}$ & Georgia, & Louisiana & $\begin{array}{c}\text { West } \\
\text { Virginia }\end{array}$ \\
\hline \multirow[t]{2}{*}{ Maximum } & 43.3 & 45.6 & 39.6 & 45.6 & 32.7 & 31.8 \\
\hline & Alaska & Wyoming & Wyoming & Wyoming & Montana & Wyoming \\
\hline \multicolumn{7}{|l|}{2016} \\
\hline Observations & 27 & 30 & 30 & 30 & 27 & 27 \\
\hline Mean & 21.21 & 22.51 & 24.13 & 24.94 & 20.81 & 18.77 \\
\hline Std. Dev. & 8.85 & 7.04 & 7.7 & 6.15 & 3.28 & 5.24 \\
\hline \multirow[t]{2}{*}{ Minimum } & 13.2 & 14.2 & 15 & 17.6 & 15.8 & 9.8 \\
\hline & Florida & $\begin{array}{l}\text { North } \\
\text { Carolina }\end{array}$ & Georgia & Mississippi & Nebraska & Nebraska \\
\hline \multirow[t]{2}{*}{ Maximum } & 61 & 44.4 & 39.6 & 40.5 & 28.2 & 36.4 \\
\hline & Alaska & Montana & $\begin{array}{l}\text { South } \\
\text { Dakota }\end{array}$ & Wyoming & Montana & Wyoming \\
\hline
\end{tabular}

states, and 3) blue versus red states, three sets of $t$-tests are run. Because suicide rates have been rising across all states since 2008, the more surprising result in the comparisons we propose for the first two sets of $t$-tests might be failure to reject the null hypothesis of no difference in the average suicide rates from one election year to the next. The third set of $t$-tests might also appear to be pointless. After all, judging from the means reported in Table 2 and Table 3, one might expect average suicide rates by age group in red states to be much greater than the corresponding average rates in blue states, in each of the three election years. Here again, failure to reject the null hypothesis of no difference between blue/red average suicide rates for any given age group might be the more surprising result.

\section{Presentation of Results}

Table 4 shows the results of the two-sample $t$-tests involving the average suicide rates for each age group in blue states alone. Despite higher average suicide rates 


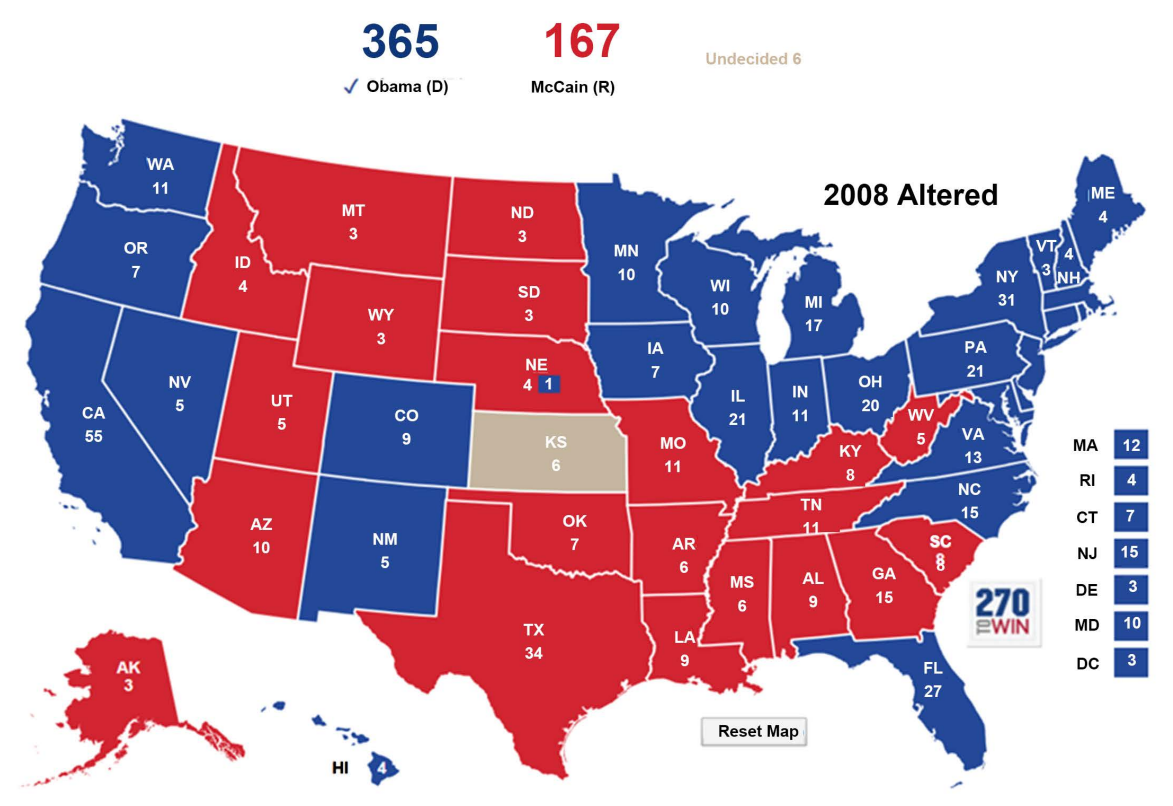

Figure 1. The 2008 election map showing the red-blue divide. Note: John McCain won all six electoral votes in Kansas (a red state). Source:

https://www.270towin.com/2008_Election/interactive_map.

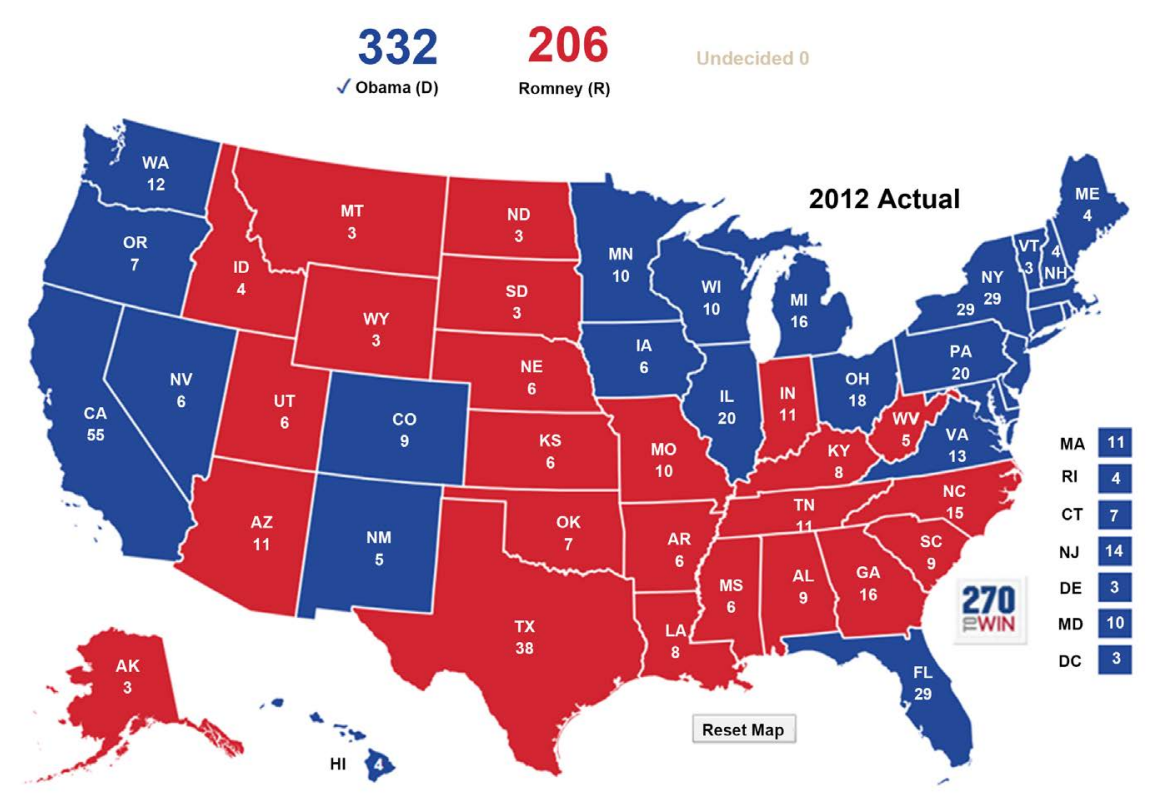

Figure 2. The 2012 election map showing the red-blue divide. Source: https://www.270towin.com/2012_Election/interactive_map.

for almost all age groups over time, in only two of the eighteen tests reported were the results statistically significant (using $\alpha=0.05$ ). In both cases, the comparisons involved the years 2008 and 2016. The higher average suicide rate in 2016 among adults between 25 and 34 years of age was discernible from the corresponding average in $2008(p=0.0402)$. And, the higher 2016 average suicide rate among adults between 55 and 64 years of age was also discernible from the corresponding average in $2008(p=0.0382)$. 


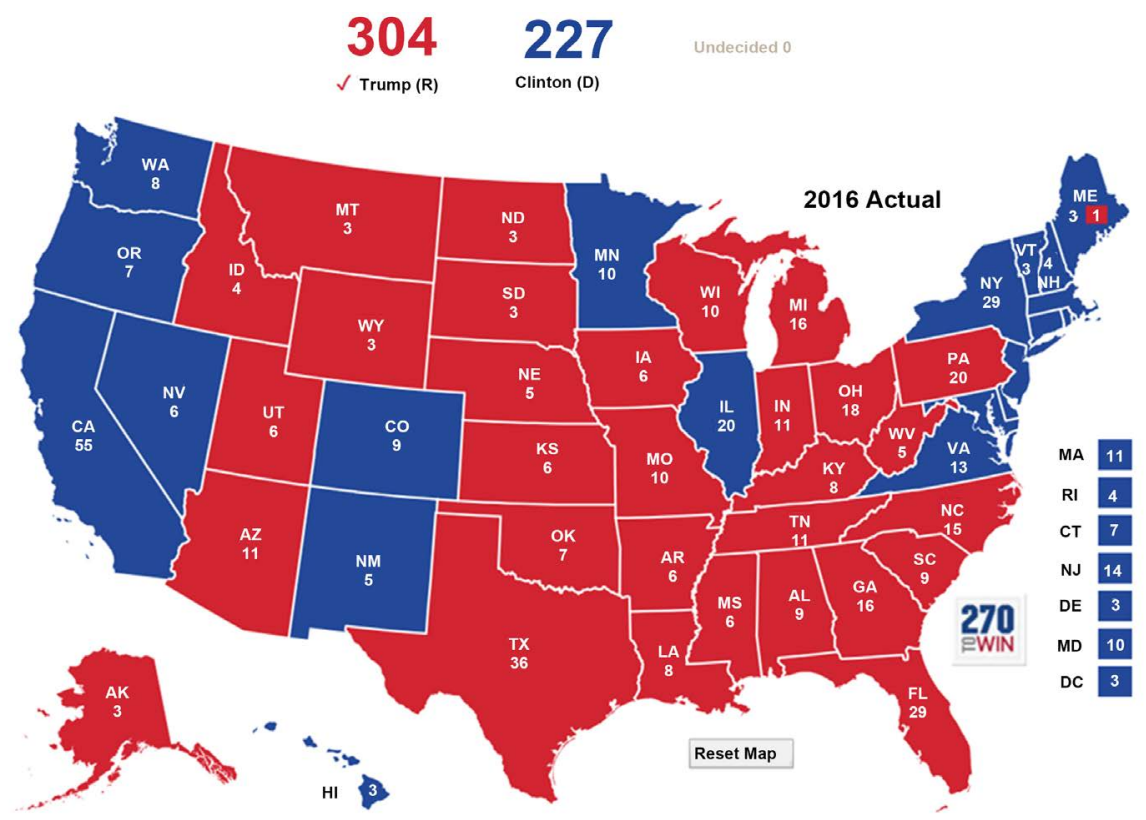

Figure 3. The 2016 election map showing the red-blue divide. Source: https://www.270towin.com/2016_Election/interactive_map.

Table 4. Average suicide rates in blue states, 2008, 2012, and 2016, by Age Group.

\begin{tabular}{|c|c|c|c|c|c|}
\hline \multicolumn{6}{|c|}{ Age Group } \\
\hline & \multicolumn{2}{|c|}{ Years } & \multicolumn{2}{|c|}{ Averages } & \multirow{2}{*}{$\begin{array}{c}\mathrm{p} \text {-value } \\
\text { on difference }\end{array}$} \\
\hline & Group 1 & Group 2 & Group 1 & Group 2 & \\
\hline & 2008 & 2012 & 13.07 & 13.92 & 0.5345 \\
\hline \multirow[t]{3}{*}{$20-24$} & 2012 & 2016 & 13.92 & 16.61 & 0.1251 \\
\hline & 2008 & 2016 & 13.07 & 16.61 & 0.0597 \\
\hline & 2008 & 2012 & 13.7 & 15.44 & 0.1749 \\
\hline \multirow[t]{3}{*}{$25-34$} & 2012 & 2016 & 15.44 & 17.18 & 0.343 \\
\hline & 2008 & 2016 & 13.7 & 17.18 & 0.0402 \\
\hline & 2008 & 2012 & 17.04 & 17.76 & 0.5813 \\
\hline \multirow[t]{3}{*}{$35-44$} & 2012 & 2016 & 17.76 & 18.17 & 0.8254 \\
\hline & 2008 & 2016 & 17.04 & 18.17 & 0.5113 \\
\hline & 2008 & 2012 & 20.25 & 21.59 & 0.4461 \\
\hline \multirow[t]{3}{*}{$45-54$} & 2012 & 2016 & 21.59 & 19.63 & 0.3081 \\
\hline & 2008 & 2016 & 20.25 & 19.63 & 0.7319 \\
\hline & 2008 & 2012 & 16.06 & 18.22 & 0.1715 \\
\hline \multirow[t]{3}{*}{$55-64$} & 2012 & 2016 & 18.22 & 19.4 & 0.4988 \\
\hline & 2008 & 2016 & 16.06 & 19.4 & 0.0382 \\
\hline & 2008 & 2012 & 14.96 & 14.69 & 0.8546 \\
\hline \multirow[t]{2}{*}{$65+$} & 2012 & 2016 & 14.69 & 17.63 & 0.1304 \\
\hline & 2008 & 2016 & 14.96 & 17.63 & 0.1822 \\
\hline
\end{tabular}

${ }^{\text {aThe }} p$-value is reported for a two-tailed two-sample $t$-test with equal variances. 
Table 5 shows the results of the two-sample $t$-tests involving the average suicide rates for each age group in red states alone. In only three of the eighteen tests reported were the results statistically significant. Two of these three results involved the years 2008 and 2016, significantly different (higher in 2016 than in 2008) for adults between 25 and 34 years of age $(p=0.0413)$ as well as for adults between 35 and 44 years of age $(p=0.0392)$. When in 2012 Mitt Romney failed to deny Barack Obama's bid for a second consecutive term, average suicide rates for adults in red states between 45 and 54 years of age spiked 16.7 percent ( $p=$ 0.0434 ) from 21.87 to 25.53 . In no other instance did average suicide rates by age group discernibly change between election years 2008 and 2012. Moreover, in no case, for either blue or red states, did average suicide rates by age group change between election years 2012 and 2016.

Table 6 shows the results of the two-sample $t$-tests involving the average suicide rates between blue and red states for each age group. In 2008, Obama defeated Republican John McCain to win the presidency. In that same year, red states reported different (discernibly higher) suicide rates among the three youngest age groups, adults between 20 and 44 years of age. The Republican Party failed to win back the White House four years later. In 2012, average suicide rates in red states were significantly different (discernibly higher) for all age groups but adults between 55 and 64 years of age. Although the Republicans won back the White House in 2016 in a victory that surprised even so-called experts, ${ }^{2}$ adults in red states were angrier and arguably more suicidal than their counterparts in blue states among adults between 25 and 54 years of age. In 2016, red rates were significantly different (discernibly higher) than blue rates among adults aged 25 to $34(p=0.0135), 35$ to $44(p=0.0105)$, and 45 to $54(p=$ $0.0043)$.

\section{Concluding Remarks}

Age-group specific suicide rates per 100,000 people are used to measure the increasingly angry political mood in U.S. presidential election years 2008, 2012 and 2016. In each election year, the U.S. is divided into blue (Democratic) and red (Republican) states. For each of six age groups in each of the three election years, average suicide rates in blue states are compared to those in red states.

Among 25- to 34-year-olds and 55- to 64-year-olds in blue states, average suicide rates in 2016 were the highest relative to the corresponding average eight years earlier. But, among blue states, average suicide rates did not significantly change from one election year to the next for any age group. In red states, adults 45 to 54 years of age were arguably most despondent (suicidal) in 2012 when Barack Obama was re-elected for a second term than they were in 2008.

In direct comparisons between blue and red states in each age group in each election year, 2012 suicide rates in red states were significantly different (much ${ }^{2}$ The night before the November 8, 2016 presidential election, statistician and editor-in-chief of ESPN's Five Thirty Eight Nate Silver gave Donald Trump only a 28.6 percent chance of winning the presidency (Silver [10]). 
Table 5. Average suicide rates in red states, 2008, 2012, and 2016, by age group.

\begin{tabular}{|c|c|c|c|c|c|}
\hline \multicolumn{6}{|c|}{ Age Group } \\
\hline & \multicolumn{2}{|c|}{ Years } & \multicolumn{2}{|c|}{ Averages } & \multirow{2}{*}{$\begin{array}{c}p \text {-value } \\
\text { on difference }\end{array}$} \\
\hline & Group 1 & Group 2 & Group 1 & Group 2 & \\
\hline \multirow{3}{*}{$20-24$} & 2008 & 2012 & 18.89 & 18.11 & 0.7644 \\
\hline & 2012 & 2016 & 18.11 & 21.21 & 0.2111 \\
\hline & 2008 & 2016 & 18.89 & 21.21 & 0.3916 \\
\hline \multirow{3}{*}{$25-34$} & 2008 & 2012 & 18.33 & 21.57 & 0.168 \\
\hline & 2012 & 2016 & 21.57 & 22.51 & 0.6559 \\
\hline & 2008 & 2016 & 18.33 & 22.51 & 0.0413 \\
\hline \multirow{3}{*}{$35-44$} & 2008 & 2012 & 20.01 & 22.92 & 0.1083 \\
\hline & 2012 & 2016 & 22.92 & 24.13 & 0.5416 \\
\hline & 2008 & 2016 & 20.01 & 24.13 & 0.0392 \\
\hline \multirow{3}{*}{$45-54$} & 2008 & 2012 & 21.87 & 25.53 & 0.0434 \\
\hline & 2012 & 2016 & 25.53 & 24.94 & 0.7389 \\
\hline & 2008 & 2016 & 21.87 & 24.94 & 0.0606 \\
\hline \multirow{3}{*}{$55-64$} & 2008 & 2012 & 18.56 & 21.27 & 0.0928 \\
\hline & 2012 & 2016 & 21.27 & 20.81 & 0.6957 \\
\hline & 2008 & 2016 & 18.56 & 20.81 & 0.078 \\
\hline \multirow{3}{*}{$65+$} & 2008 & 2012 & 17.1 & 18.34 & 0.3466 \\
\hline & 2012 & 2016 & 18.34 & 18.77 & 0.7538 \\
\hline & 2008 & 2016 & 17.1 & 18.77 & 0.2698 \\
\hline
\end{tabular}

${ }^{a}$ The $p$-value is reported for a two-tailed two-sample $t$-test with equal variances.

Table 6. Difference in suicide rates between blue and red states, 2008, 2012, and 2016, by age group.

\begin{tabular}{|c|c|c|c|c|}
\hline \multicolumn{5}{|c|}{ Age Group } \\
\hline & \multicolumn{3}{|c|}{ Averages } & \multirow{2}{*}{$\begin{array}{c}\mathrm{p} \text {-value } \\
\text { on difference }\end{array}$} \\
\hline & Year & Blue & Red & \\
\hline & 2008 & 13.07 & 18.89 & 0.0094 \\
\hline \multirow[t]{3}{*}{$20-24$} & 2012 & 13.92 & 18.11 & 0.0312 \\
\hline & 2016 & 16.61 & 21.21 & 0.0768 \\
\hline & 2008 & 13.7 & 18.33 & 0.0061 \\
\hline \multirow[t]{3}{*}{$25-34$} & 2012 & 15.44 & 21.57 & 0.0035 \\
\hline & 2016 & 17.18 & 22.51 & 0.0135 \\
\hline & 2008 & 17.04 & 20.01 & 0.0377 \\
\hline \multirow[t]{3}{*}{$35-44$} & 2012 & 17.76 & 22.92 & 0.0033 \\
\hline & 2016 & 18.17 & 24.13 & 0.0105 \\
\hline & 2008 & 20.25 & 21.87 & 0.3241 \\
\hline \multirow[t]{3}{*}{$45-54$} & 2012 & 21.59 & 25.53 & 0.0425 \\
\hline & 2016 & 19.63 & 24.94 & 0.0043 \\
\hline & 2008 & 16.06 & 18.56 & 0.1097 \\
\hline \multirow[t]{3}{*}{$55-64$} & 2012 & 18.22 & 21.27 & 0.063 \\
\hline & 2016 & 19.4 & 20.81 & 0.2771 \\
\hline & 2008 & 14.96 & 17.1 & 0.1761 \\
\hline \multirow[t]{2}{*}{$65+$} & 2012 & 14.69 & 18.34 & 0.008 \\
\hline & 2016 & 17.63 & 18.77 & 0.5486 \\
\hline
\end{tabular}

The $p$-value is reported for a two-tailed two-sample $t$-test with equal variances. 
higher) than they were in blue states for every age group but adults 55 to 64 years of age.

Future research might examine the spread in age-group specific average suicide rates between blue and red states in earlier election years or the off-election years before and after each election. The detailed age-specific death CDC data are available back to 1999. Readers might take issue with using suicide as a measure of polarization and despondency. Less drastic measures of the nation's mood, though, may be more difficult to quantify and hence may be less meaningful in making interpersonal comparisons among adults living in different states.

\section{Conflicts of Interest}

The author declares no conflicts of interest regarding the publication of this paper.

\section{References}

[1] American Foundation for Suicide Prevention, N.p. https://afsp.org/about-suicide/suicide-statistics/

[2] Cohn, N. (2013) The Surge in Suicides Has Nothing to Do With Marriage or Religion. The New Republic, N.p.

https://newrepublic.com/article/113253/increase-suicide-us-not-due-marriage-or-re ligion-decline

[3] Hamermesh, D. and Soss, N.M. (1974) An Economic Theory of Suicide. Journal of Political Economy, 82, 83-98. https://doi.org/10.1086/260171

[4] Lewandusky, M.S. and Pope, J.C. (2011) Red States vs. Blue States: Going beyond the Mean. The Public Opinion Quarterly, 75, 227-248. https://doi.org/10.1093/poq/nfr002

[5] Aisch, G., Pearce, A. and Yourish, K. (2016) How Large Is the Divide between Red and Blue America? The New York Times, N.p.

https://www.nytimes.com/interactive/2016/11/04/us/politics/growing-divide-betwe en-red-and-blue-america.html

[6] Jacobson, L. (2013) Are States Deepening the Nation's Red-Blue Divide? N.p. http://www.governing.com/topics/politics/gov-states-deepening-nations-red-blue-d ivide.html

[7] Centers for Disease Control and Prevention Suicide Death Rates for Ages 20 - 24 by State in Years 2008, 2012, 2016:

https://wonder.cdc.gov/controller/saved/D76/D38F594

Suicide Death Rates for Ages 25 - 64 in Ten-Year Age Groups by State in Years 2008, 2012, 2016:

https://wonder.cdc.gov/controller/saved/D76/D38F595

Suicide Death Rates for Ages 65 or over by State in Years 2008, 2012, 2016:

https://wonder.cdc.gov/controller/saved/D76/D38F596

[8] Presidential Election Maps 2008 Presidential Election Map. https://www.270towin.com/2008_Election/interactive_map

2012 Presidential Election Map.

https://www.270towin.com/2012_Election/interactive_map

2016 Presidential Election Map. 
https://www.270towin.com/2016_Election/interactive_map

[9] Bishop, B. (2008) The Big Sort. Mifflin, Houghton.

[10] Silver, N. (2016) Who Will Win the Presidency? N.p. https://projects.fivethirtyeight.com/2016-election-forecast/ 